\title{
Romania. World Library of Folk and Primitive Music
}

Compilé et édité par Alan Lomax, Rounder Records (2001)

\section{Victor A. Stoichiță}

\section{(2) OpenEdition}

\section{Journals}

Édition électronique

URL : http://journals.openedition.org/ethnomusicologie/823

ISSN : 2235-7688

\section{Éditeur}

ADEM - Ateliers d'ethnomusicologie

\section{Édition imprimée}

Date de publication : 1 novembre 2002

Pagination : 216-218

ISSN : 1662-372X

Référence électronique

Victor A. Stoichiță, «Romania. World Library of Folk and Primitive Music », Cahiers d'ethnomusicologie [En ligne], 15 | 2002, mis en ligne le 11 janvier 2012, consulté le 19 avril 2019. URL : http://

journals.openedition.org/ethnomusicologie/823

Ce document a été généré automatiquement le 19 avril 2019.

Tous droits réservés 


\title{
Romania. World Library of Folk and Primitive Music
}

\author{
Compilé et édité par Alan Lomax, Rounder Records (2001)
}

\author{
Victor A. Stoichiță
}

\section{RÉFÉRENCE}

Romania. World Library of Folk and Primitive Music. Compilé et édité par Alan Lomax, vol. XVII. Rounder Records ROUN 1759 (2001). Enregistrements choisis dans les archives de l'Institut de Folklore de Bucarest par Tiberiu Alexandru. Livret (en anglais) : Tiberiu Alexandru et Speranţa Rădulescu.

1 Depuis quelques années, l'ensemble de la «Columbia World Library of Folk and Primitive Music » est en passe d'être réédité. Sept disques l'ont été pour le moment, celui-ci étant l'un des plus récents. Initialement paru en 1963 sous le titre The Folk Music of Rumania (Columbia KL 5799), il avait rapidement gagné une certaine notoriété, mais s'était retranché depuis au fond de quelques rares discothèques. Il s'agit d'une compilation "panoramique», dont l'objectif est de permettre "un tour d'horizon de la musique paysanne roumaine, représentatif de toutes les aires culturelles, catégories musicales et styles historiques et régionaux " (préface de la seconde édition). Destinée à un large public, elle constitue une introduction remarquable par la valeur documentaire et (parfois) esthétique de ses enregistrements, accompagnés d'un livret concis mais informatif.

2 La sélection initiale a été choisie dans le fonds de l'Institut de Folklore de Bucarest par Tiberiu Alexandru, à la demande d'Alan Lomax. Soit dit en passant, on chercherait vainement le nom de ce dernier à la page "crédits » en fin du livret: si la plupart des compilations de la collection bénéficient de sa contribution à la notice et éventuellement aux enregistrements, ce n'est manifestement pas le cas de celle-ci. La plupart des pièces ont été recueillies par différents chercheurs dans les années 50, avec deux exceptions 
notables, enregistrées par Constantin Brăiloiu en 1934 et 1936 (plages 14 et 32). Une partie de la sélection fut publiée sur le 33 tours, accompagnée d'une « introduction à la musique populaire roumaine » par Tiberiu Alexandru, qui signait également le commentaire des plages.

Cette réédition, dont le maître d'œuvre est SperanŢa Rădulescu, introduit plusieurs changements. Cinq pièces du 33 tours, jouées par des ensembles folkloriques, ont été remplacées par cinq autres, plus "traditionnelles", également issues de la sélection envoyée à Lomax par Alexandru. Une très belle ballade (Iovan Iorgovan, plage 3), dont seul un extrait figurait sur le 33 tours, est ici présentée en entier. Enfin, l'ordre des plages a été légèrement modifié et le livret a été revu et complété.

Il s'ouvre à présent sur une « préface à la seconde édition » (4 p.) par SperanŢa Rădulescu, qui commente le disque d'origine et les changements survenus lors de sa réédition. Suit l'« introduction » de Tiberiu Alexandru (2 p.), telle qu'elle figurait sur le 33 tours. Il s'agit essentiellement d'une description des catégories dans lesquelles les enregistrements sont classés, et sur lesquelles il nous faudra revenir dans un instant. Enfin, le commentaire des plages (18 p.) est celui de l'original, revu et augmenté par la rééditrice. Outre les informations indispensables (nom des interprètes, date de l'enregistrement, etc.), il resitue brièvement chaque pièce dans son contexte géographique et historique, en attirant l'attention sur ses caractéristiques les plus saillantes. Le commentaire des plages s'achève souvent par une brève présentation des interprètes, ce qui permet d'éviter l'impression d'anonymat fréquente sur d'autres compilations. Ce faisant, le livret parvient à rester concis, tout en échappant aux simplifications abusives. Le cas échéant, les textes sont transcrits en roumain et en anglais. Des photos aèrent la lecture et une carte des enregistrements permet de localiser rapidement leur provenance.

Les plages sont groupées en huit sections : « ouverture », « musique rituelle ", « musique de danse ", « répertoire pastoral », « doine (chants longs) », « ballades et chants épiques », "chants lyriques», "lăutari (musiciens professionnels, urbains ou ruraux)». Cette présentation est risquée et suscite inévitablement des questions. Par exemple : pourquoi toutes les pièces jouées par des lăutari ne sont-elles pas regroupées dans la section qui leur est consacrée (on en trouve également parmi les « chants rituels ", les "danses » et les "ballades») ? Pourquoi une catégorie "musique pastorale", qui comprend une seule pièce (l'histoire du « berger qui a perdu ses moutons »), jouée qui plus est par un prêtre? Quant à la section « ouverture ", où trône un unique " signal de printemps " joué au tulnic (sorte de cor des alpes), sa fonction est éventuellement esthétique mais il eût sans doute été plus clair de l'inclure dans le "répertoire pastoral», dont elle est un exemple légitime. Au-delà de ces incohérences, le découpage est gênant car il tend à présenter ces catégories comme des répertoires aux frontières bien établies, ce qui n'est vraiment le cas ni pour les chercheurs ni pour les musiciens. Il est vrai que, « ouverture » mise à part, ce classement n'est pas une innovation du disque. Il est fréquent dans la littérature de spécialité de l'époque et n'est d'ailleurs pas dénué d'une certaine pertinence. On aurait simplement apprécié une définition plus approfondie et peut-être un commentaire, l'introduction de Tiberiu Alexandru étant plutôt laconique à son sujet.

6 Les trente-cinq enregistrements proviennent de l'ensemble du territoire, illustrent les principaux styles musicaux et offrent un panorama organologique très complet. L'objectif du disque est donc atteint. Toutefois, la musique des minorités ethniques reste dans l'ombre, ce en quoi le titre est quelque peu trompeur. On peut cependant atténuer cette critique pour un disque conçu dans les années 60 , d'autant plus que la préface de la 
réédition prévient le lecteur de cette restriction. Une autre pratique courante à l'époque était de couper les pièces dont on pensait qu'elles risquaient de lasser l'auditeur. C'est pour cela que douze des enregistrements ne sont présentés qu'à titre d'extrait, ce qui est certes un peu frustrant mais reste dans des proportions tolérables. Le choix des pièces a clairement été guidé par des considérations esthétiques et plusieurs musiciens hors pair figurent ainsi sur la compilation. On les remarque à l'écoute, mais également à la lecture du livret qui mentionne, le cas échéant, le caractère exceptionnel de leurs aptitudes. Les quelques « remaniements » folkloriques - plutôt bien choisis d'ailleurs - sont également repérés dans le texte. Leur présence s'explique par la volonté panoramique du disque (sans eux le tableau eût été incomplet), mais leur proportion élevée sur le 33 tours était également due aux contraintes idéologiques de l'époque et elle a été réduite sur le $\mathrm{CD}$. Au final, le disque laisse une impression variée, multicolore, abordant parfois des genres rarement entendus ailleurs.

7 On remarque par exemple le chant «de passage» (de petrecut, plage 8), destiné à accompagner les morts vers l'autre monde. Deux chœurs féminins chantent en antiphonie. Chacun s'élève et retombe doucement sur la tonique, la prolongeant en guise de bourdon pour l'autre. C'est l'un des seuls exemples de polyphonie - plus précisément de tuilage - dans le chant roumain. Celle-ci est fréquente par contre chez les Aroumains, une population roumanophone disséminée entre l'Albanie et la Mer Noire, d'où provient le chant de la plage 31. Il est remarquable d'ailleurs que cette communauté soit représentée sur le disque, car elle est absente de la plupart des autres compilations de musique roumaine.

8 La doina du pays de l'Oaş (plage 18) est quant à elle un bel exemple du genre décrit sous le nom de horă lungă(«chant long») par Béla Bartók (Rumanian Folk Music, vols II et V. La Haye, 1967-73). Chaque vers, chanté recto tono sur la note la plus haute, est suivi d'une longue descente mélismatique. Celle-ci est ornée et rythmée grâce à une technique vocale caractéristique, une sorte de coup de glotte qui peut être plus ou moins marqué.

La «suite de danŢ pour danser » (plage 14), enregistrée par Constantin Brăiloiu, illustre elle aussi un genre très particulier, circonscrit à une petite région du nord de la Roumanie (le Pays de l'Oaş). Plus que les deux violons qui accompagnent la fête, ce sont les « hurlements » (Tîpurîturi) des danseurs qui retiennent ici l'attention. Ils ne s'alignent pas vraiment sur la pulsation, leur échelle reste floue, mais ils jouent néanmoins un rôle central dans ce répertoire, influant par exemple sur l'accordage des violons ${ }^{1}$.

Le disque présente aussi certains instruments rarement entendus ailleurs, comme la tilinca (plage 10), qui est une longue flûte sans conduit ni trou de jeu (un simple tuyau, en somme). On la trouve parfois sous sa forme « domestiquée » - avec un conduit qui facilite l'insufflation - mais peu de musiciens sont capables de maîtriser celle qui est jouée ici.

11 Enfin, on notera plusieurs pièces qui apparaissent également sur d'autres disques, mais figurent sur celui-ci dans des interprétations particulièrement savoureuses. C'est le cas par exemple du «chant de la mariée » (plage 9), qui est un «classique» du rituel de mariage, et des compilations de musique roumaine. Interprété par un excellent chanteur, accompagné par un Tुambal (petit cymbalum)et un violon, il illustre admirablement le style des lăutari de Clejani, bien avant qu'ils ne deviennent célèbres sous le nom de « Taraf de haïdouks ».

12 Cette compilation contient de nombreux enregistrements remarquables, qu'on pourrait commenter pendant longtemps. Le disque et son livret sont en cela une réussite car, loin 
de simplifier la présentation, ils ouvrent sans cesse de nouvelles perspectives. Certains genres sont devenus rares de nos jours, d'autres se sont modifiés, mais ce tour d'horizon reste pour les néophytes une excellente introduction à la musique roumaine et pour les autres, un rappel peut-être utile de sa richesse et de sa variété passées.

\section{NOTES}

1. Cf. Jacques Bouët,Bernard Lortat-Jacob et SperanŢa Rădulescu : A tue-tête. Nanterre : Société d'ethnologie, 2002. 